\title{
Barriers to Weight Management: Perspectives of Plus-Sized Black Women
}

\author{
Jolene Lynn, RN, PhD ${ }^{1}$, Peggy Ward-Smith, RN, PhD $^{2}$ \\ Jolene Lynn, School of Nursing and Health Studies, University of Missouri, Kansas City, MO 64108 USA. \\ School of Nursing and Health Studies, University of Missouri - Kansas City2464 Charlotte Street Kansas City, MO \\ 64086
}

\begin{abstract}
Obesity, the number one health condition in the United States, is especially prevalent among Black, middle-aged, urban women. The purpose of this qualitative study was to describe the experience of being plussized, from the perspective of these individuals. Interview data were obtained from nine participants, using phenomenological research methods and focus group format. Data were analyzed into significant statements, which were placed into these. Three themes emerged from these data: (1) health consequences, (2) barriers toward weight management, and (3) motivators toward weight management. These individuals knew that being plus-sized had health consequences, and could describe specific barriers and motivators that would be helpful. Including this information in any planned intervention will increase involvement, and improve the health status of this population. (122 words)
\end{abstract}

Keywords: Black women, plus-sized, weight management, phenomenology

\section{INTRODUCTION}

Obesity is the number one health condition in the United States (U.S.) (Odgen, Carroll, Kit, \&Flegal, 2014). At present, more than one-third of all U.S. adults are obese. While 39.5\% of all individuals between the ages of 40 and 50 years are obese, Non-Hispanic Blacks have the highest age-adjusted rate at $47.8 \%$ (Odgen et al., 2014). The combination of being middle-aged, and of non-Hispanic background compounds the potential for being obese. Health consequences of obesity, as identified by the Centers for Disease Control and Prevention (CDC) include heart disease, stroke, type 2 diabetes, and certain types of cancer (2015). Exercise has been identified by Healthy People 2020 (U.S. Department of Health and Human Services, n.d.) as one intervention that prevents weight gain or facilitates weight loss. Yet, opportunities to engage in meaningful exercise, for the urban-dwelling, middle-aged, black female are few. The demographics of these individuals reveal that employment opportunities tend to be bluecollar, fitness centers are not close by, transportation is limited, and leisure time is sparse. The purpose of this study was to explore the challenges of weight management, specifically from the perspective of the Black, middle-aged, urban female. The aim of this study was to gain an understanding of the phenomena of physical activity for the plussized, urban dwelling, Black woman. Using qualitative research methods provided a mechanism to include the importance of culture and to gain an understanding of a healthy body image, from the Black women's perspective. According to the State of Obesity (2105), Blacks and urban dwellers are underserved populations, thus little is known about their experiences. Phenomenology obtains data directly from a primary source, the individual directly familiar with the experience under investigation. Using these data to develop interventions will assure that they are culturally respectful and reflective of the unique needs of these individuals.

\section{STUDY METHOD}

Qualitative research, using a phenomenological method, is appropriate when little is known about the topic under investigation, the experience has yet to be conceptualized, or the research aims to describe the experience (Polit\&Beck, 2012). The results of phenomenological research allows the researcher to understand the meaning of the experience, from the social, cultural, and historical context. This methodology aligns with the study aim. Data were collected using focus groups. This interactive data collection method allows participants to interact with one another, and provides a 'normal conversation' approach toward data collection. (Kvale\&Brinkmann, 2009) Thus, the participants were free to provide details, with other participants adding to the content, or providing an opposing point of view.

\footnotetext{
${ }^{1}$ Corresponding Author: lynnjj@umkc.edu
} 
Institutional Review Board (IRB) approval from the University where the researcher is a doctoral student was secured prior to any research activity. Once study approval was attained, snowball sampling techniques were used to recruit study participants. The initial participant was an acquaintance of one of the researchers and familiar with the study. While not desiring to be a participant, she was willing to identify other individuals.

Study participation was limited to Black females who self-reported being between the ages of 40 and 65 years, residing in an urban setting, and plus-size. Plus-size was defined as wearing clothing that is labeled plus-size, or between the sizes of 12 and 24. Body Mass Index (BMI) was not calculated, nor was weight reported.

Three focus groups were held in a private conference room at the local acute care facility. Each focus group was audiotaped and followed the IRB-approved semi-structured interview guide. The global research question was "please tell me, in youropinion, if or how your weight influences your life." Probing questions were then used to identify barriers, which limited or prevented physical activity, and activities which assisted in the ability to partake in physical activity. Each participant was afforded the opportunity to clarify any statement, and provide additional information pertinent to the topic.

Both investigators were present for all interviews, and maintained separate field notes. Thoughts, perceptions, body language, and intensity of expression were captured in these field notes. Data collection commenced after the investigators introduced themselves, verbally explained the study and written consent was provided. Each interview was guided by one of the investigators, and began with the global research question. Once all study data were obtained, and prior to ending the audio-recording, each participant was ask specific demographic questions. These questions were used only to describe the study population.

\section{RESULTS}

There were nine study participants. While this number may appear small, Speziale and Carpenter (2007) recommend between 6 and 12 as appropriate for qualitative research, or until data saturation is achieved. Data saturation, meaning no new information was heard, occurring during the third focus group. Each participant completed all study activities, and no adverse effects, associated with study participation, were reported. Each group session lasted between 30 and 50 minutes (mean $=42)$.

Demographically, the participants self-described themselves as Non-Hispanic Black, between the ages of 43 to 65 years $($ mean $=59$ ). Four of the participants $(44 \%)$ described their relationship status as married; three $(33 \%)$ declined to disclose their relationship status and two (22\%) stated they were unmarried (single, widowed). Six participants (66\%) reported the presence of adult children in their household; these same six participants (66\%) stated they were employed part-time. One participant stated they were working full-time; the remaining two participants describing their employment status as retired or unemployed. Self-described weight-related health conditions included joint problems and hypertension (66\%) and diabetes (44\%).

Once all data collection were complete, the interview data were transcribed verbatim and triple checked for accuracy. After the written transcripts were validated by both investigators, the find and replace option in WORD was used to replace all identifying information (names) with pseudonyms. Then, the audio-tapes were destroyed, in compliance with IRB regulations at the University. HyperResearch(Hesse-Biber, Kinder, \& Dupuis, 1990) was used to manage these data; analyses was guided by Colaizzi's (1978) phenomenological steps. Significant statements and themes were identified by each investigator individually, with consensus used for final determination. Themes were then labeled to reflect the content.

\section{THEMES}

\subsection{Health Consequences Associated With Obesity}

Health consequences associated with obesity was known by each participant. All participants knew that obesity was linked to a multitude of health conditions, as well as a decreased life span. Eight participants (89\%) described a family member whose health was negatively impacted by obesity, which resulted in less-than-optimal health outcomes, despite treatment. Obesity was described by each participant as a familiar trait, with many family members being overweight. Six participants (66\%) could not remember not being 'large', which was described as being 'big boned' or 'robust.'

\subsection{Barriers toward weight management}

Barriers, as described by these participants, encompassed five variables. These included (1) time, (2) money, (3) motivation, (4) safety, and (5) physical limitations. Time limitations were described as "I simply do not have the 
time to cook healthy meals, or exercise." "My family responsibilities come first, and after that, the day is gone." Money limitations included both the cost of any gym membership and the expense of healthy food options. "For me, the cost of either the local YMCA or a personal trainer are just not feasible. I have to figure it out for myself, and I have no idea what is right." "There are actually two barriers that effect healthy food choices - one the cost of fresh fruits and vegetables is high and two is the lack of their availability locally." Motivation, when identified as a barrier, was described as a lack of personal attributes and the unavailability of an individual to provide the extra incentive necessary to maintain a healthy lifestyle. The statement "I am just too tired at the end of a day to think about anything but resting" was made by three (33\%) participants. Other motivation-related barriers included "a lack of anyone to walk with" or "the weather (too hot or too cold) makes being outdoors not appealing" and "I can lose a little weight, but maintaining that loss is impossible." The safety of the residential area was mentioned by seven (78\%) participants as a barrier to any time of outside activity. "There are stray dogs in this neighborhood - that alone prevents me from being outside." "There seem to always be people I do not recognize in my neighborhood and that makes me leery of taking a walk." The physical limitations associated with weight management were described as temporary "I broke my ankle a while back, and that foot does not seem strong enough to do much walking." "I know this is silly, but I am afraid of falling. My [family member] fell and it seems as if they were never the same."

\subsection{Motivators toward weight management}

Interventions that were helpful, or perceived as helpful, included scheduling exercise time, using the churchsupported exercise area, and having peer support. Scheduling time for cooking or exercising did not guarantee that the activity would occur, but it did increase the possibility. "I try to have an idea for dinner before I leave work and have the foods necessary ready to go. That prevents snack and junk food from happening." "At my church, there is an exercise area. There is a waitlist to belong, because it is cheap - like $\$ 2.00$ each time you go. But you have to go at least three times a week to stay in the program. If you do it for one month, the next month is free. It is hard, but that makes it do-able." "For me, scheduling a time to walk or cook with my neighbor makes things happen. I do not want to let her down, so I make an effort."

\section{LiMitationS}

Limitations associated with this study include the use of one geographical location as the study site. Environmental limitations associated with a Midwestern location may not be generalizable to other urban locations. This particular urban setting has a limited public transportation system, and has been described as a grocery desert. These variables may not be present in other locations. The use of snowball sampling techniques may have inadvertently skewed the study population. Each participant was familiar with the study site which was the local acute health facility. This may have resulted in participants who were more familiar with their health than other study populations. The investigators are White, middle-class women, which might have inhibited any participant from completely expressing their perspective. The content of the interviews, and the field notes of the investigators do not seem to reflect this limitation. In fact, the field notes and reflections of the investigators indicate that, in their opinion, these participants seemed elated that someone was interested in obtaining their perspective.

\subsection{Implications for Practice}

Individual culture, life-style, and living arrangements impact weight management. While weight has genetic and metabolic influences, physical activity has been demonstrated to be effective in preventing or reversing obesity. The results of this study demonstrate that knowledge alone will not result in physical activity. Each participant in this study was aware of the health consequences of obesity. Yet, there are real, tangible barrier that prevent them from participating in routine physical activity. These individuals were able to articulate specific interventions that would improve their ability to become active. Any intervention developed or implemented aimed at increasing physical activity needs to include data, obtained directly from the intended population. The results of this study describe a population that, while plus-size, have knowledge and suggestions that will improve their ability to exercise. The women in this study appear motivated to improve their health and understood that exercise is integral as part of a plan to increase healthy behaviors.

\section{Conclusions}

Obesity is a complex health problem, with metabolic and genetic influences (Obesity Society, 2010). Personal willpower and access to interventions are modifiable variables that have been linked to weight control (Fletcher, Hanson, Page, \& Pine, 2011). The participants in this study, which represent blue collar, urban dwelling, middleages, Black females, had the knowledge necessary to link being plus-sized to health consequences yet the majority 


\section{American Research Journal of Nursing, Volume 1, Issue 1, 2015}

ISSN 2379-2922

of the women did not have a plan to implement healthier behaviors. Time, cost, motivation, safety, and physical problems were identified as barriers to improve activity levels.

\section{REFERENCES}

[1] Center for Disease Control and Prevention (CDC) (2015). Obesity Facts and Figures. Retrieved from:http://www.cdc.gov/obesity/data/adult.html

[2] Colaizzi, P.F. (1978). Psychological research as the phenomenologist views it. In R.S. Valle \&M. King (Eds.), Existentialphenomenological alternatives in psychology (Chapter 3). New York: Oxford University Press

[3] Fletcher, B., Hanson, I., Page, N., \& Pine, K. (2011). FIT-Do something different: a new behavioral program forsustained weight loss. Swiss Journal of Psychology, 70(1), 25-34.

[4] Hesse-Biber, S., Kinder, S., \& Dupuis, P.R. (1990). HyperRESEARCH 3.7.2 Computer Software, Researchware, Inc.

[5] Kvale, S., \&Brinkmann, S. (2009). Interviews: Learning the craft of qualitative research interviewing(2nd ed.). Thousand Oaks, CA: Sage Publications

[6] Obesity Society (2010). Obesity, bias, and stigmatization. Retrieved from: http://www.obesity.org/resources-for/obesitybias-and-stigmatization.htm

[7] Ogden, C.L., Carroll, M.D., Kit, B.K., \&Flegal, K.M. (2014). Prevalence of childhood and adult obesity in theUnited States, 2011-2012. Journal of the American Medical Association 311(8), 806-814. doi: 10.1001/jama.2014.732

[8] Polit, D.F., \& Beck, C.T. (2012). Nursing research: Generating and assessing evidence for nursing practice (9th ed.). Philadelphia, PA: Lippincott, Williams \& Wilkins

[9] Speziale H.J. S. \& Carpenter, D.R. (2007). Qualitative research in nursing. Philadelphia: Lippincott Williams \&Wilkins.

[10] State of Obesity (2015). Obesity prevention in black communities. Retrieved from: http://stateofobesity.org/disparities/blacks/U.S. Department of Health and Human Services (n.d.). Office of Disease Prevention and Health

[11] Promotion. Healthy People 2020. Washington, DC, Retrieved from: https://www.healthypeople.gov/2020/topicsobjectives/topic/physical-activity 